\title{
ISOMORPHISM THEOREMS ON QUASI MODULE
}

\author{
SANDIP JANA \\ Department of Pure Mathematics \\ University of Calcutta \\ 35, Ballygaunge Circular Road, Kolkata-700019, India \\ e-mail: sjpm@caluniv.ac.in \\ AND \\ Supriyo MAZumder \\ Department of Mathematics \\ Adamas University \\ Barasat - Barrackpore Road, Jagannathpur, Kolkata, India \\ e-mail: supriyo88@gmail.com
}

\begin{abstract}
A quasimodel is an algebraic axiomatisation of the hyperspace structure based on a module. We initiated this structure in our paper [2]. It is a generalisation of the module structure in the sense that every module can be embedded into a quasi module and every quasi module contains a module. The structure a quasimodel is a conglomeration of a commutative semigroup with an external ring multiplication and a compatible partial order. In the entire structure partial order has an intrinsic effect and plays a key role in any development of the theory of quasi module. In the present paper we have discussed order-morphism which is a morphism like concept. Also with the help of the quotient structure of a quasi module by means of a suitable compatible congruence, we have proved order-isomorphism theorem.
\end{abstract}

Keywords: module, quasi module, order-morphism, congruence, quotient.

2010 Mathematics Subject Classification: 08A99, 13C99, 06F99.

\section{REFERENCES}

[1] T.S. Blyth, Module Theory: an Approach to Linear Algebra (Oxford University Press, USA).

[2] S. Jana and S. Mazumder, An associated structure of a Module, Revista de la Academia Canaria de Ciencias XXV (2013) 9-22. 
[3] S. Mazumder and S. Jana, Exact sequence on quasi module, South. Asian Bull. Math. 41 (2017) 525-533.

[4] S. Jana and S. Mazumder, Quotient structure and chain conditions on quasi modules, Buletinul Academiei De Stiinie A Republicii Moldova Mathematica 2(87) (2018) $3-16$.

Received 4 December 2018

Revised 16 January 2019

Accepted 23 January 2019 